Vom UMgang Mit Literatur UND Literaturgeschichte 


\section{Vom Umgang mit Literatur und Literaturgeschichte}

\section{Positionen und Perspektiven nach der »Theoriedebatte»}

\section{Herausgegeben von}

Lutz Danneberg und Friedrich Vollhardt in Zusammenarbeit mit Hartmut Böhme und Jörg Schönert

J. B. Metzlersche Verlagsbuchhandlung Stuttgart 


\section{Mit freundlicher Unterstützung der Deutschen Forschungsgemeinschaft}

Die Deutsche Bibliothek - CIP-Einheitsaufnahme

Vom Umgang mit Literatur und Literaturgeschichte : Positionen und Perspektiven nach der "Theoriedebatte" / hrsg. von Lutz Danneberg und Friedrich Vollhardt in Zusammenarbeit mit Hartmut Böhme und Jörg Schöne. - Stuttgart : Metzler, 1992

ISBN 978-3-476-00788-9

ISBN 978-3-476-03386-4 (eBook)

DOI 10.1007/978-3-476-03386-4

NE: Danneberg, Lutz [Hrsg.]

\section{ISBN 978-3-476-00788-9}

Dieses Werk einschließlich aller seiner Teile ist urheberrechtlich geschützt. Jede Verwertung außerhalb der engen Grenzen des Urheberrechtsgesetzes ist ohne Zustimmung des Verlages unzulässig und strafbar. Das gilt insbesondere für Vervielfältigungen, Übersetzungen, Mikroverfilmungen und die Einspeicherung und Verarbeitung in elektronischen Systemen.

(C) 1992 Springer-Verlag GmbH Deutschland

Ursprünglich erschienen bei J. B. Metzlersche Verlagsbuchhandlung und Carl Ernst Poeschel Verlag GmbH in Stuttgart 1992 


\section{Inhalt}

Vorwort

\section{Sektion 1}

Einleitung. Interpretation und Argumentation: Fragestellungen der

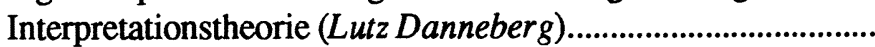

Dieter Freudlieb: Literarische Interpretation. Angewandte Theorie oder soziale Praxis?

Klaus Blaudzun und Heinz-Jürgen Staszak: Dialektik der Interpretation. Zu Voraussetzungen des methodologischen Nachdenkens über die literaturwissenschaftliche Interpretation

Rainer Hegselmann: Logik und Dialektik. Zum Projekt einer Theorie des Argumentierens

Christoph Lumer: Handlungstheoretisch erklärende Interpretationen als Mittel der semantischen Bedeutungsanalyse.

\section{Sektion 2}

Einleitung. Auslegung und Deutung literarischer Texte: Prinzipien wissenschaftlicher Bewertung und Begründung (Friedrich Vollhardt).

Andreas Kamlah: Verstehen und Rekonstruieren. Zur Theorie der Geisteswissenschaften.

Gerhard Pasternack: Zur Rationalität der Interpretation.......................... 149

Karl Eibl: Sind Interpretationen falsifizierbar? ........................................ 169

Werner Stribe: Über Kriterien der Beurteilung von Textinterpretationen

Harald Fricke: Methoden? Prämissen? Argumentationsweisen! Überlegungen zur Konkurrenz wissenschaftlicher Standards in der Literaturwissenschaft 


\section{Sektion 3}

Einleitung. Konzepte und Exempel der Interpretation literarischer und philosophischer Texte (Hartmut Böhme).

Gottfried Gabriel: Zur Interpretation literarischer und philosophischer Texte

Reinhard Brandt: Von der Hermeneutik zur Interpretation 251

Wolfgang Proß: Historische Methodik und philologischer Kommentar

Liliane Weissberg: Literatur als Repräsentationsform. Zur Lektüre von Lektüre.

Gerhard Kurz: Vieldeutigkeit. Überlegungen zu einem literaturwissenschaftlichen Paradigma

\section{Sektion 4}

Einleitung. Möglichkeiten und Probleme einer Integration von Literaturgeschichte in Gesellschafts- und Kulturgeschichte (Jörg Schönert)

Reiner Wild: Literaturgeschichte - Kulturgeschichte - Zivilisationsgeschichte

Hans-Ulrich Mohr: Von der Regelpoetik zur Wirkungsästhetik?

Der Wandel des englischen Literatursystems im

18. Jahrhundert in funktionsgeschichlicher Sicht

Friederike Meyer: Diskurstheorie und Literaturgeschichte. Eine systematische Reformulierung des Diskursbegriffs von Foucault ......

Claus-Michael Ort: Vom Text zum Wissen. Die literarische Konstruktion sozio-kulturellen Wissens als Gegenstand einer nicht-reduktiven Sozialgeschichte der Literatur.

Gert Sautermeister: Literarhistorisches Interpretieren. Am Beispiel Gottfried Kellers. 


\section{Vorwort}

Der Ruf nach einem Paradigmawechsel hat in der Literaturwissenschaft seine bedrohliche, aber auch seine faszinierende Wirkung verloren. Darin mag etwas Beruhigendes liegen, zumal die Ankündigung neuer Verfahren der Werkbetrachtung oft genug in Versprechen mündete, die uneingelöst blieben. Wo gegenwärtig über die Voraussetzungen und Bedingungen der literaturwissenschaftlichen Forschung nachgedacht wird, geschieht dies aus einem betont heuristischen oder pragmatischen Interesse. Die weit gestreuten Ansätze stehen nicht mehr in einem Gesprächszusammenhang, dem man vor zwei Jahrzehnten den stattlichen Titel einer $>$ Theoriedebatte $<$ verliehen hat. Theoretische und methodische Begründungsansprüche sind kaum mehr Gegenstand der Diskussionen, die in der fachlichen Öffentlichkeit geführt werden. Erkennbar wird das Bemühen, jene am Ende der sechziger Jahre erhobene Forderung nach einer wissenschaftlichen - und politischen - Rechtfertigung des Faches als abgeschlossenen Teil der eigenen Geschichte zu begreifen. Der Wissenschaftsbetrieb hat inzwischen neue Umgangsformen und Kriterien für die Beurteilung von Leistung und Erfolg hervorgebracht. Vor diesem Hintergrund erscheint es heute als störend, wenn man auf der Frage nach der theoretischen Legitimität derjenigen Konzeptionen besteht, die gegenwärtig das Erscheinungsbild der Disziplin bestimmen.

Die Evidenz, mit der eine an traditionell hermeneutischen Vorstellungen orientierte Auslegungspraxis noch immer zu überzeugen vermag, läßt leicht über den prinzipiellen Relativismus nebeneinander bestehender Interpretationsverfahren hinwegsehen. Man hat gelernt, den Pluralismus, ja auch die Beliebigkeit der Untersuchungsmethoden zu akzeptieren. Mitunter werden sie zur Entlastung zitiert, will man nicht die eigene Praxis auf ihre (wissenschaftliche) Verbindlichkeit hin befragt wissen. Die Kluft zwischen einem sich stets neu stellenden theoretischen Innovationsdruck und der exponierten Theoriefeindlichkeit wird dabei häufig über eine erneuerte, anderen Disziplinen entlehnte Begrifflichkeit geschlossen. Terminologische Freibeuterei suggeriert das Vorhandensein eines methodischen Rahmens, der unausgefüllt bleibt, solange die mitgesetzten Bedingungen nicht auf die eigene Tätigkeit bezogen werden.

Angesichts der hier nur angedeuteten Diskontinuität und letztlich enttäuschenden Inkonsequenz, mit der über theoretische Fragen in den immer weiter gezogenen Grenzen des Fachs diskutiert worden ist, wird von verschiedenen Seiten auf die Entwicklung empirischer oder medienwissenschaftlicher (Teil-) Disziplinen gedrängt, die kaum mehr an die Wissenschaft anknüpfen, die in 
herkömmlicher Weise allein Texte untersucht. Diesen Zustand der Literaturwissenschaft kann man beklagen oder begrüßen - in jedem Fall läßt er sich analysieren.

Die hier zusammengefaßten Beiträge bildeten die Grundlage des Ersten Internationalen Hamburger Kolloquiums »Zu Problemen der Literaturinterpretation undLiteraturgeschichtsschreibung", das im März 1989 in Hamburg stattgefunden hat. Sie liefern eine Bestandsaufnahme inzwischen weitgehend abgeschlossener Reflexionen zur Interpretationstheorie und Literaturhistoriographie und verbinden diese Standortbestimmung mit der Vorstellung weiter entwickelter Forschungsansätze. Schwerpunkte bilden (1) der Bereich der professionellen Textinterpretation und, auf einer zweiten Ebene, die ihr zugrundeliegenden Verstehensprozesse; neben die klassischen Fragen der Textauslegung tritt (2) die Historizität des Gegenstandes und damit die Praxis der Literaturgeschichtsschreibung, deren theoretische Grundannahmen anhand einiger ihr neuerdings zugeordneter Arbeitsfelder (Diskursanalyse, funktionale Systemtheorie) untersucht werden. An der Hamburger Tagung haben teilgenommen:

\footnotetext{
Klaus Blaudzun (Sektion Sprach- und Literaturwissenschaften, Universität Rostock)

Reinhard Brandt (Fachbereich Philosophie, Universität Marburg)

Karl Eibl (Trier, jetzt Institut für Deutsche Philologie, Universität München)

Dieter Freundlieb (Griffith University, Nathan, Brisbane/Australien)

Harald Fricke (Seminar für deutsche Literatur, Universität Fribourg/Schweiz)

Gottfried Gabriel (Fachgruppe Philosophie, Universität Konstanz)

Rainer Hegselmann (Fachbereich 10-Philosophie, Universität Bremen)

Jochen Hörisch (Seminar für Deutsche Philologie, Universität Mannheim)

Andreas Kamlah (Fachbereich Kultur- und Geowissenschaften, Universität Osnabrück)

Klaus Laermann (Fachbereich Germanistik, Freie Universität Berlin)

Christoph Lumer (Fachbereich Kultur- und Geowissenschaften, Universität Osnabrück)

Friederike Meyer (München)

Hans-Ulrich Mohr (Fakultät für Linguistik und Literaturwissenschaften, Univ. Bielefeld)

Claus-Michael Ort (München, jetzt Institut für Literaturwissenschaft, Universität Kiel)

Gerhard Pasternack (Zentrum Phil. Grundlagen der Wissenschaften, Universität Bremen)

Roland Posner (Institut für Linguistik, Technische Universität Berlin)

Wolfgang Proß (Deutsches Seminar, Universität Bern/Schweiz)

Werner Strube (Institut für Philosophie, Universität Bochum)

Liliane Weissberg (Baltimore, jetzt University of Pennsylvania, Philadelphia/USA)

Hans-Georg Werner (Wissenschaftsbereich Deutsche Literatur, Universität Halle)

Reiner Wild (Saarbrücken, jetzt Fakultät für Geistes- und Sozialwissenschaften, TH Karlsruhe)
}

Wir haben uns darum bemüht, auch die Beiträge jener Eingeladenen in diesen Band aufzunehmen, die aus unterschiedlichen Gründen die Teilnahme an dem Tagungsgespräch absagen mußten. Gerhard Kurz und Gert Sautermeister konnten unserer Bitte nachkommen.

Alle Vorlagen sind zuvor an die Teilnehmer verschickt worden, so daß die Tagung ganz im Zeichen der Diskussion stand. Wichtige Ergebnisse des Gesprächs sind von den Autoren in die Druckfassung der Beiträge eingearbeitet worden; die den Beiträgen vorangestellten Einleitungen pointieren Ergebnisse des Diskussionsverlaufs. 
Die Herausgeber danken Christian Weller für seine Arbeit an den Diskussionsprotokollen, Wilhelm Schernus für die Redaktion und dem Metzler-Verlag für die Übernahme des Kolloqium-Bandes in sein Programm. Die Deutsche Forschungsgemeinschaft gewährte einen Druckkostenzuschuß und trug zusammen mit der Hamburger Behörde für Wissenschaft und Forschung die Finanzierung des Kolloquiums. Wir danken beiden Institutionen für die hilfreiche Unterstützung.

Hamburg, im März 1991

Lutz Danneberg, Friedrich Vollhardt 